\title{
Estudio clínico de los implantes dentales Naturactis en postextracción dental
}

\section{Clinical study of naturactis dental implants postextraction dental procedures}

\author{
RIPOLLÉS DE RAMÓ́N J* \\ GÓMEZ FONT R** \\ BASCONES-ILUNDAIN C*** \\ BASCONES-ILUNDAIN J**** \\ BASCONES-MARTÍNEZ A*****
}

\begin{abstract}
Ripollés de Ramón J, Gómez Font R, Bascones-Ilundain C, BasconesIlundain J, Bascones-Martínez A. Estudio clínico de los implantes dentales naturactis en postextracción dental. Av Periodon Implantol. 2013; 25, 3: 135-142.
\end{abstract}

\section{RESUMEN}

Introducción: Los implantes inmediatos postextracción tienen un éxito variable según diferentes autores entre un $92,7 \%$ y $98,0 \%$. Su principal indicación es la sustitución de dientes susceptibles de extracción. Su ventaja principal es la de acortar el tiempo de tratamiento rehabilitador y evitar una segunda cirugía. Como inconveniente destaca el requerir, generalmente, técnicas de regeneración tisular guiada.

Material y método: 60 implantes Naturactis de Euroteknika (Euroteknika Groupe, Sallanches - Francia) fueron colocados de forma inmediata postextracción siguiendo un protocolo clínico de actuación. 33 pacientes fueron incluidos en este estudio en base a unos criterios de inclusión. Se analizaron variables clínicas (edad, sexo y hábitos tóxicos), quirúrgicos (torque de inserción, evaluación periodontal, estabilidad con Ostell) radiológicos (pérdida ósea crestal). Todos ellos fueron analizados 10 meses vista. Se evitaron las descargas mucoperiósticas en todos los casos.

Resultados: Los implantes colocados obtuvieron un torque de inserción $>35 \mathrm{Nm}$ tras su colocación así como unos valores >63 ISQ de Ostell. El protocolo quirúrgico fue satisfactorio por los operadores. 4 implantes del total fracasaron de forma temprana ( $<1$ mes). Se constató ausencia de pérdida ósea crestal en los primeros 6 meses de su colocación. La cicatrización y postoperatorio fue satisfactoria con ausencia de complicaciones.

PALABRAS CLAVE: Implantes dentales, implantes inmediatos, postextracción, carga inmediata.

\section{SUIMIMARY}

Introduction: Dental implants places in dental fresh sockets, have a variable success according to different authors, between $92,7 \%$ and $98 \%$. Its principle indication is to substitute teeth admitting dental extraction. Its principle advantage is a shorter time of treatment up to loading of the prosthesis, also it avoids a second surgery and improves the healing process. As an inconvenient we remark, the need for guided tissue regeneration.

Material and method: 60 dental implants (Naturactis from Euroteknika Groupe, Sallanches - Francia) were placed immediate after dental extraction with a clinical protocol. 33 patients were included in this study, based on some inclusion criterias. We analyzed clinical aspects like (age, gender, toxic

\section{*}

versidad Complutense de Madrid. España.

** Profesor Ciencias de la Salud. Universidad Europea de Madrid. España.

*** Profesor Asociado UCM. Departamento de Medicina y Cirugía Bucofacial. Facultad de Odontología.

**** Profesor Asociado UCM. Departamento de Odontología Conservadora. Facultad de Odontología.

***** Catedrático del Departamento de Medicina y Cirugía Bucofacial. Facultad de Odontología. Universidad Complutense de Madrid. España. 
habits), surgical aspects (insertion torque, periodontal evaluation, ostell stability measure), radiology (crestal alveolar resorption). All these cases were analyzed during 10 months. Mucoperiostical flaps were avioded in all cases.

Results: All dental implants placed, achieved at least $35 \mathrm{NM}$ after placed, as well a >63 ISQ measured with Ostell. The surgical protocol was approved by surgeons. 4 of all the implants placed failed in the early stages ( $<1$ month). We verify no crestal bone loss in the first 6 months after the implants were placed. Healing and post surgical procedures were correct without any complication described.

KEY WORDS: Dental implants, inmediate dental implants, inmediate load.

Fecha de recepción: 1 de septiembre de 2013.

Fecha de aceptación: 30 de octubre de 2013.

\section{INTRODUCCIÓN}

La colocación de implantes inmediatos postextracción dental es una técnica desarrollada en los últimos 20 años (1-8). Esta técnica surge en base a las crecientes necesidades de acortar los tratamientos implantológicos por un lado y, por otro lado, evitar la atrofia de las estructuras alveolares y mantener la mayor integridad posible de tejido duro y blando en el proceso alveolar, tendente a disminuir en volumen después de la extracción dental. Esta técnica se encuentra íntimamente relacionada con la aplicación de técnicas de regeneración tisular, para compensar los espacios existentes entre el lecho aleveolo-implantológico y el propio implante evitando así la migración de células epiteliales a esta zona y, por tanto, interfiriendo en el proceso de osteointegración (9-14). Este procedimiento tiene sus limitaciones, indicaciones y contraindicaciones como cualquier otro, siendo necesario un volumen óseo residual mínimo tanto por vestibular/palatino del implante, como del hueso sano apical a éste $(15,16)$.

La colocación de implantes dentales postextracción ha supuesto una técnica más entre los numerosos avances de la implantología actual y son numerosos los artículos publicados que demuestran la predictibilidad de la técnica como la seguridad de la misma con unas tasas de éxito similares a los casos quirúrgicamente tratados de forma diferida, tal y como publica Chen $(17,18)$.

El grado de satisfacción de los pacientes tras aplicar esta técnica es evidente, puesto que acortamos sustancialmente el tiempo de tratamiento. Esta técnica debe realizarse de una manera meticulosa y de una forma precisa e indicada, puesto que no está exenta de complicaciones, en especial, cuando ésta no es realizada correctamente, siendo la causa más común de fracaso la infección del proceso alveolar, que conduce, en la mayoría de los casos, a una pérdida del implante dental.

\section{HIPÓTESIS Y OBJETIVOS}

Los implantes Naturactis tienen una morfología y un diseño macro y micromecánico que favorece la posición y la estabilización del implante en el alveolo dental, especialmente postextracción, de forma similar a otros procedimientos convencionales. Las características del protocolo quirúrgico simplifica el procedimiento favoreciendo la instalación del implante, y por lo tanto, favoreciendo el mínimo traumatismo del tejido periodontal y haciendo que el sistema permita un mínimo traumatismo periodontal.

Los objetivos del presente estudio fueron los siguientes:

- Evaluación del grado de estabilidad primaria tras la colocación del implante.

- Evaluación del grado del éxito del implante Naturactis en el periodo de 6-12 y 18 meses tras su colocación.

- Análisis del tejido periodontal circundante a la colocación del implante.

- Estudiar el grado de aparición de periimplantitis tras la colocación de los implantes.

- Evaluar el grado de satisfacción del profesional con el manejo clínico del implante así como de su protocolo quirúrgico.

\section{MATERIAL Y MÉTODO}

Se realizó un estudio clínico longitudinal prospectivo con una muestra poblacional de 60 implantes dentales Naturactis en un total de 33 pacientes, en base a unos criterios de inclusión y exclusión médicos y dentales.

El tamaño muestral inicialmente fue de 60 implantes Naturactis colocados postextracción. La selección de la muestra fue una aproximación puramente clínica, a fin de evaluar estadísticamente los datos obtenidos y 
analizarlos desde un punto de vista clínico, con el fin de aumentar la muestra en próximos estudios, si éste fuera positivo.

Los pacientes firmaron un consentimiento informado del proceso médico así como la información de la inclusión en un estudio clínico de investigación.

Los pacientes partícipes del estudio fueron seleccionados de forma voluntaria de clínicas privadas de Madrid.

El estudio clínico fue aprobado por el comité ético de evaluación e investigación del Hospital Clínico San Carlos de Madrid el 11 de Julio de 2012 con el código CP CI 12/268E.

Se establecieron unos criterios de inclusión y exclusión al estudio que se describen a continuación.

- Criterios de inclusión:

- Sexo: Indiferente.

- Rango de edad: 18-65 años.

- Conservación del estado de salud general.

- Criterio clínico de extracción de un diente del intervalo 15-25, 35-45 en ausencia de signos de infección dentoperiodontal así como de los criterios e indicaciones propias de la técnica de colocación de los implantes inmediatos postextracción, entre las que destacamos:

- Al menos $1 \mathrm{~mm}$ de hueso por vestibular o palatino en el proceso alveolar donde va colocado el implante dental.

- Hueso sano mínimo apical al diente extraído y por tanto en la futura zona de colocación del implante de al menos 3-5 mm.

- Ausencia de infección.

Tratamiento con amoxicilina clavulánico 875/125 mg cada 8 horas durante una semana, comenzando el tratamiento 2 días antes de la intervención.

- Criterios de exclusión:

- Pacientes polimedicados (más de tres fármacos) o fármacos que influyan en el metabolismo de la regulación ósea (bifosfonatos).

- Alergias de cualquier tipo.

- Pacientes embarazadas o en periodo de lactancia.

- Ingesta de fármacos que puedan alterar los parámetros de coagulación $\mathrm{y} / \mathrm{vit} \mathrm{K}$.

- Consumo de más de 10 cigarrillos/día.

- Síndromes discrásicos.

- Procesos psiquiátricos.

- Alcoholismo o ingesta de alcohol en periodo inferior a 7 días.
En relación a los criterios establecidos para la colocación del implante, se establecieron como necesarios: la presencia de uno o más dientes en la arcada superior o inferior entre las posiciones comprendidas entre ambos premolares susceptibles de ser extraídos por distintas causas (enfermedad periodontal, fractura radicular, reabsorciones radiculares externas o internas, imposibilidad de tratamientos endodónticos o fracasos de los mismos).

\section{Metodología y técnica quirúrgica}

Una vez incluido el paciente objeto de estudio se procedieron a los siguientes pasos:

- Consentimiento informado.

- Ortopantomografía.

- Confección de un bloque de godiva (se grabarán las iniciales del paciente).

- Realización de una radiografía periapical ajustada con el bloque de godiva confeccionado (Figura 1).

- Fotografía inicial.

\section{Técnica quirúrgica}

El procedimiento quirúrgico aplicado para la extracción será el de una cirugía lo más atraumática posible, conservando la estructura ósea periodontal con la mayor integridad posible. Se realizará un cureteado alveolar a fin de encontrar un sangrado del lecho alveolar. Una vez realizada la exodoncia, se procedió a la colocación del implante dental Naturactis de conexión interna siguiendo la secuencia de fresado del fabricante. En el caso de existir una discrepancia en-

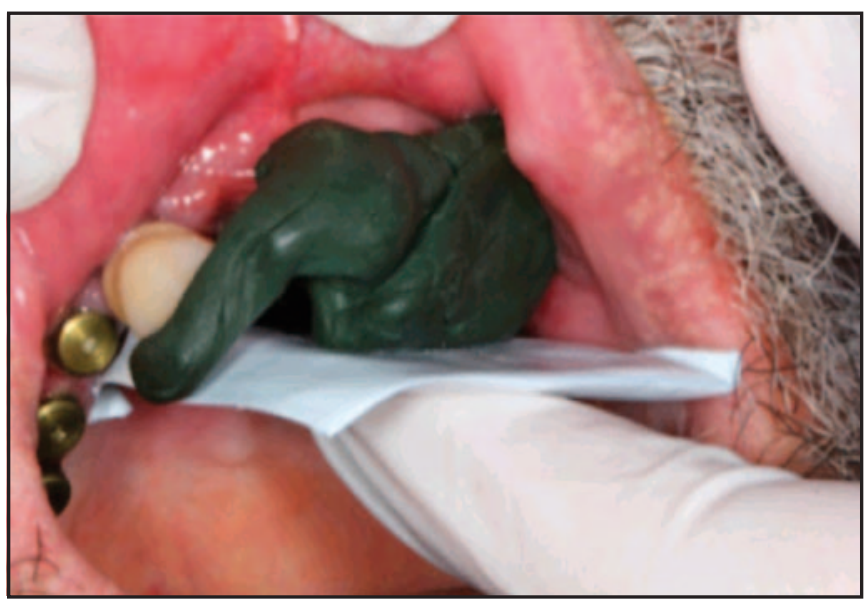

Fig. 1: Confección del bloque de godiva. 
tre el implante y el espacio alveolar superior a $1 \mathrm{~mm}$ la colocación del implante será realizada de forma inmediata a la exodoncia y con un torque mínimo de 35 Nmc. En caso de no ser alcanzada esta estabilidad, no entraría el caso dentro del estudio. Una vez colocado el implante, mediremos la estabilidad del mismo medida con Ostell y anotado su valor en valores ISQ (Figura 2).

El implante se coloca con un tornillo de cicatrización, de altura mínima necesaria para que éste se encuentre expuesto y permita posteriores mediciones con Ostell.

La ausencia de estabilidad primaria superior a $35 \mathrm{Nm}$, la existencia de infección alrededor del espacio alveolar, así como de granuloma o fístula actuarían como criterios de exclusión absolutos del procedimiento.

Se requiere la existencia de un mínimo de $3 \mathrm{~mm}$ de hueso alveolar sano apical a la colocación del implante así como un mínimo de $1 \mathrm{~mm}$ de espesor óseo en las tablas vestibular y palatina (Figuras 3 y 4 ).

Una vez colocado el implante medimos tanto el torque de inserción como la estabilidad con Ostell y realizamos una radiografía periapical con técnica paralela, utilizando el bloque de godiva inicialmente confeccionado a fin de poder controlar la evolución crestal con la misma proyección en las sucesivas visitas. Por último, realizamos una fotografía similar a la inicial en este momento, con el implante colocado y con el tapón de cicatrización colocado (Figura 5).

Los pacientes se trataron con amoxicilina/ácido clavulánico 500/125 mg a razón de 1 comprimido cada 8 horas durante una semana así como fármacos analgé-

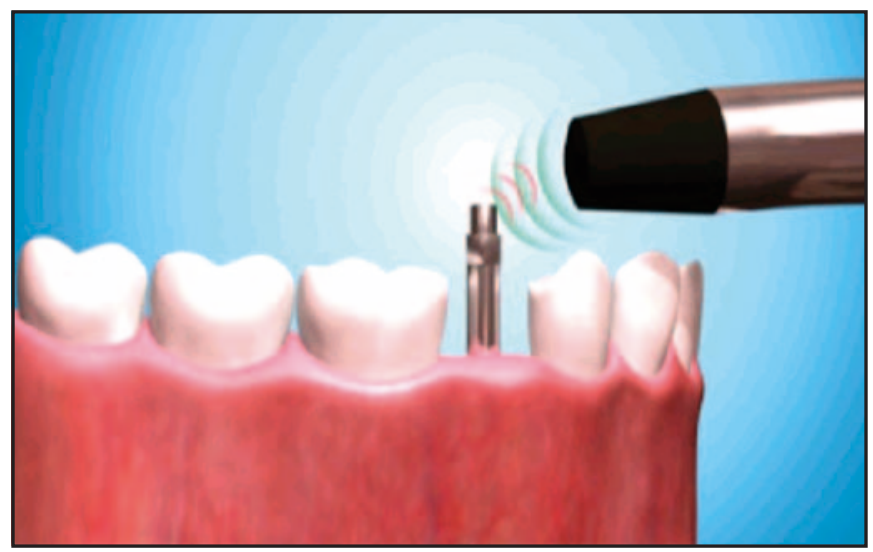

Fig. 2: Medición estabilidad con Ostell y smartpeg.

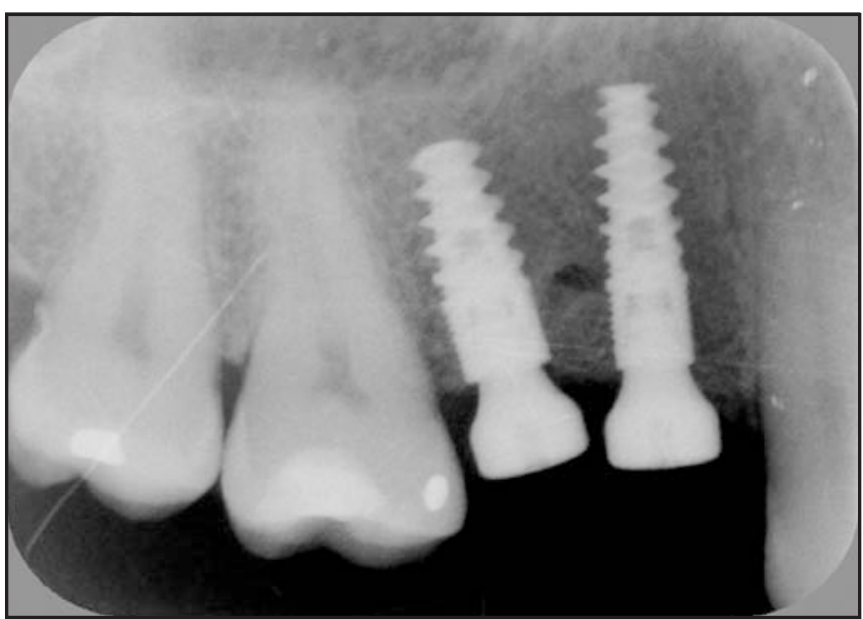

Fig. 3: Radiografía periapical con bloque de godiva.

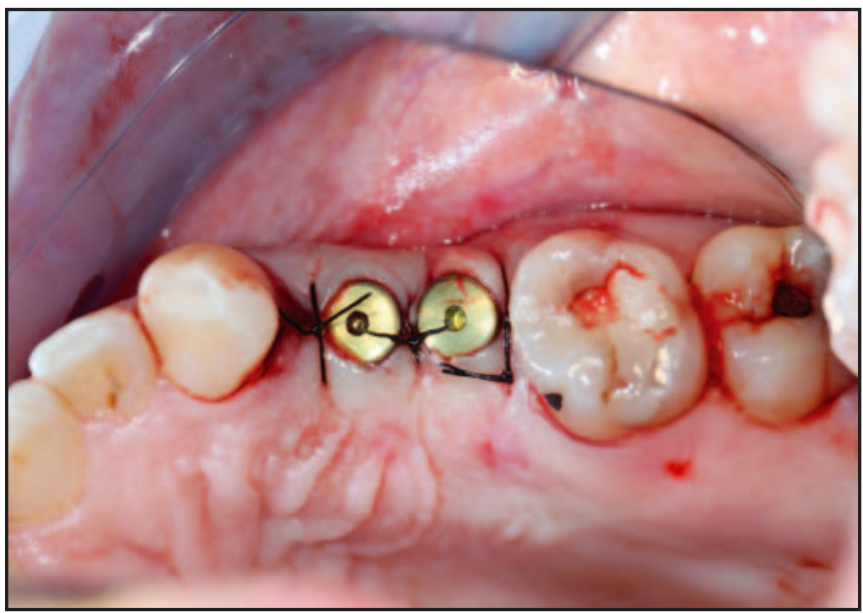

Fig. 4: Colocación de implantes naturactis postextracción con tornillo de cicatrización.

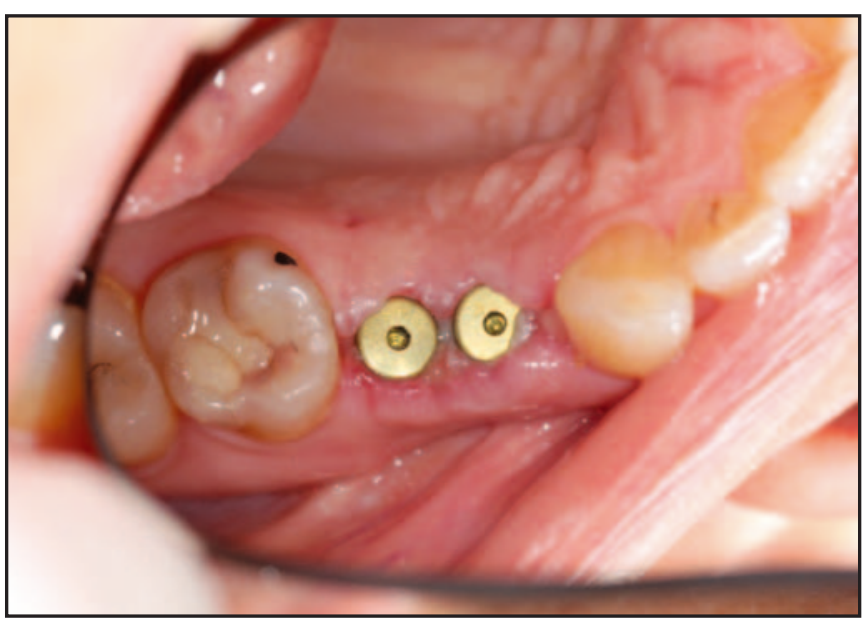

Fig. 5: Fotografía a los 7 días de la intervención. 
sicos antiinflamatorios durante las primeras $24-48 \mathrm{~h}$. Se recomendó el uso de clorhexidina $0,20 \%$ de forma tópica durante 15 días.

A los 7 días se cita al paciente para retirar la sutura, realizar una medición de la estabilidad con Ostell, realizar una radiografía periapical. Estos procedimientos se repiten a los 2,4 y 6 meses, momento en el que se comienza la rehabilitación del caso.

\section{RESULTADOS}

En relación a las variables clínicas, las clasificaremos en variables relacionadas con el sexo, la edad y los hábitos tóxicos. Un total de 60 implantes dentales Naturactis se colocaron en 33 pacientes, de los cuales 12 eran varones y 21 , mujeres (Gráfico 1). Más del 50\% de los pacientes tenían una edad superior a los 54 años; el siguiente grupo de edad más prevalente fue el del rango comprendido entre los 30 y 42 años (Gráfico 2).

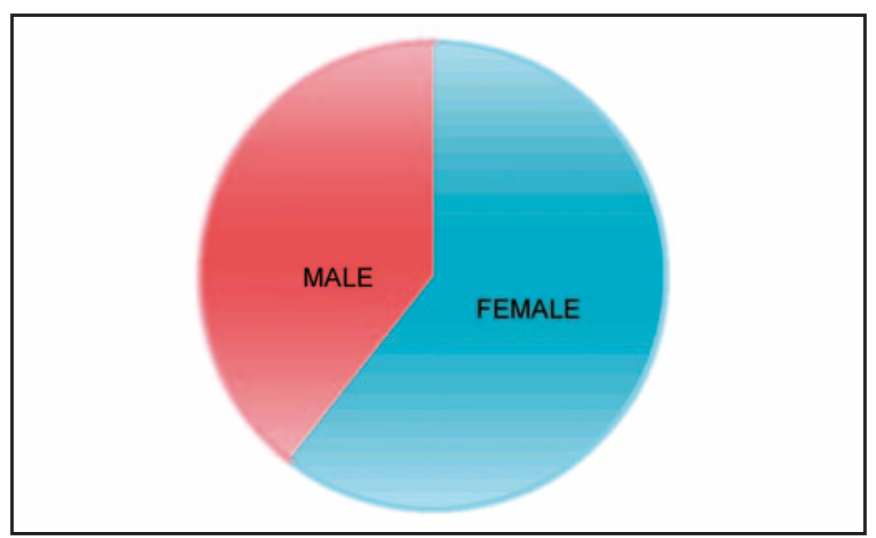

Gráf. 1: Distribución de la población por sexos.

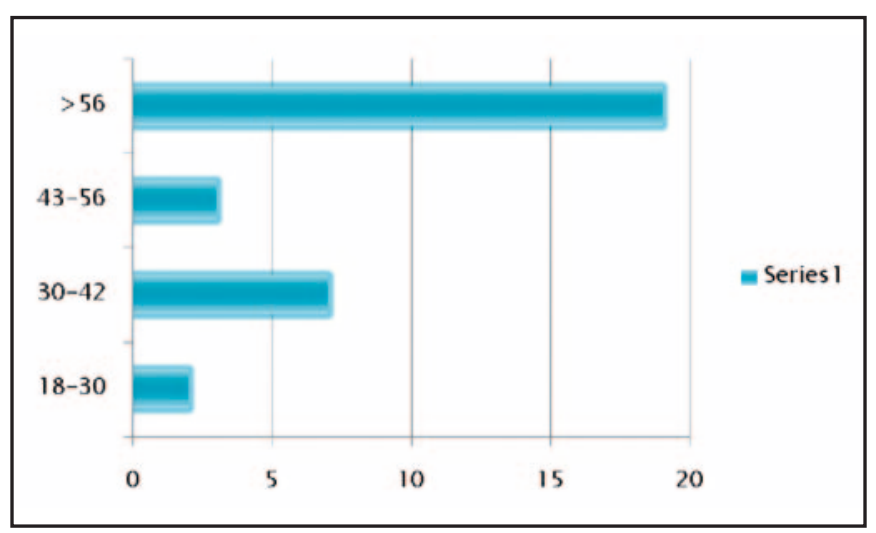

Gráf. 2: Distribución de la población por rangos de edad.
Un $80 \%$ de los implantes colocados fueron implantados a nivel maxilar mientras el $20 \%$ a nivel mandibular (Gráfico 3). Un 7\% de nuestra población refirió fumar (<10 cigarrillos/día) (Gráfico 4). De los 60 implantes colocados, 4 fracasaron y fueron retirados; de los 4 implantes fracasados, 3 lo hicieron de forma precoz o inmediata (<30 días) y uno de ellos lo hizo a los 70 días (Gráfico 5). Asimismo destacar que el fracaso de 3 de ellos fue a nivel maxilar y el otro a nivel mandibular. A los tres meses del fracaso se repitió la colocación de los 4 implantes y todos fueron cargados, con éxito, a los seis meses.

El diámetro más utilizado de los implantes fue de plataforma regular de $4 \mathrm{~mm}$ con más de la mitad de los casos seguido de la plataforma de diámetro $3 \mathrm{~mm}(35 \%)$ y el resto de plataforma ancha de $5 \mathrm{~mm}$ (Gráfico 6).

En relación a la estabilidad primaria de los implantes colocados fue, en todos ellos (100\%), superior a $35 \mathrm{Nm}$ y la estabilidad medida con Ostell tuvo unos rangos ini-

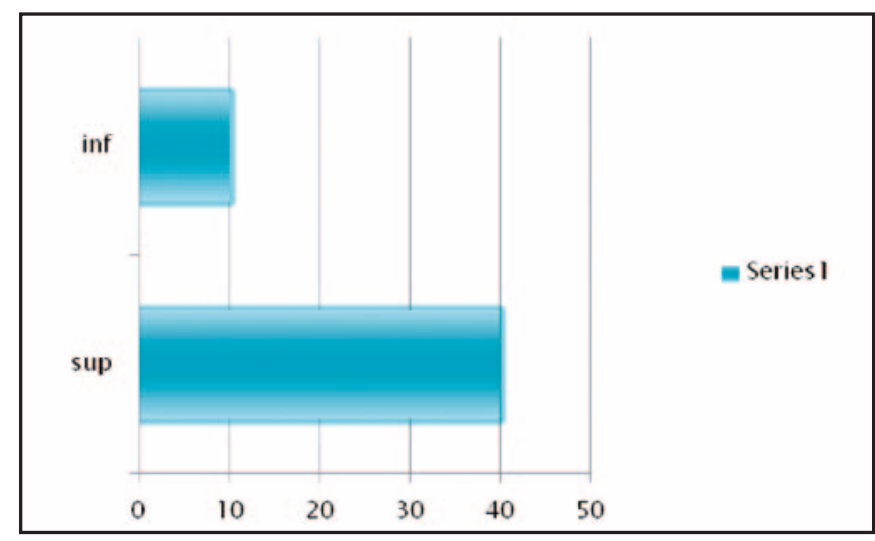

Gráf. 3: Distribución de la arcada de colocación de los implantes.

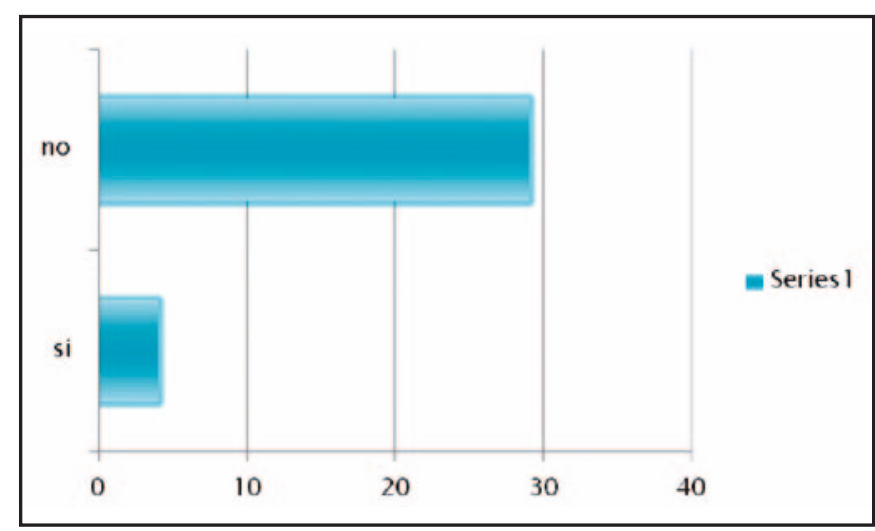

Gráf. 4: Hábitos tóxicos. Fumadores. 


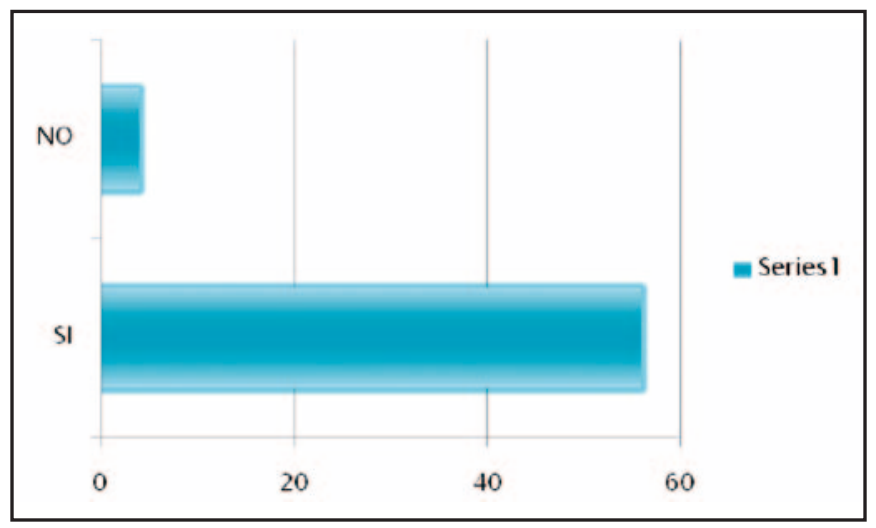

Gráf. 5: Tasa de osteointegración de los implantes. Ocho meses.

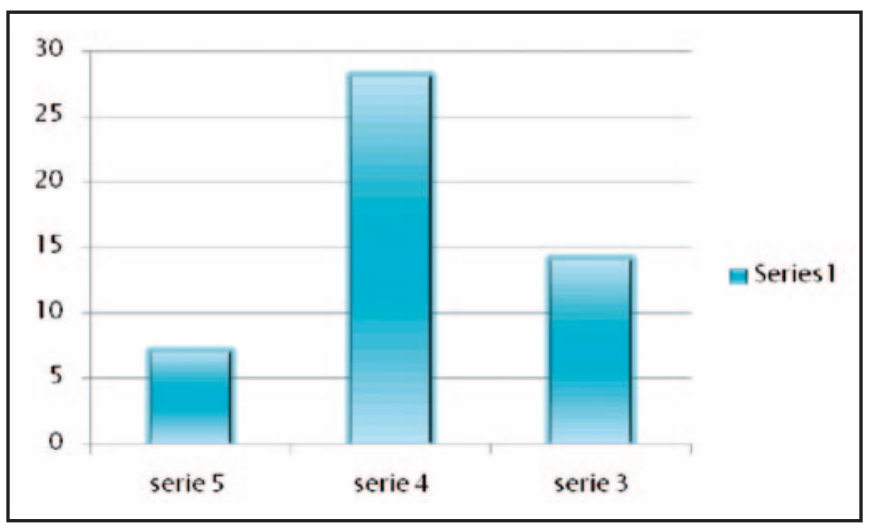

Gráf. 6: Diámetro de los implantes utilizados.

ciales comprendidos entre 64 ISQ y 92 ISQ; la tendencia de estos valores fue a disminuir en la medición del segundo mes de forma leve, volviendo a incrementar dicho valor en la medición del cuarto mes y se estabilizó en el sexto mes sin variación significativa en estas dos últimas.

Cabe destacar que, a 12 meses vista del estudio, no hubo fracaso de la carga protésica en ninguno de los casos tratados. Todos fueron dados de alta con éxito.

\section{DISCUSIÓN}

La ventaja más importante de los implantes colocados de forma inmediata postextracción, es la menor duración del tiempo total del tratamiento; también caben destacar otras, tales como la disminución del número de procedimientos quirúrgicos, así como la menor exposición a fármacos en una única sesión (1, 5, 19-21).
Por el contrario, en múltiples casos nos vemos obligados a utilizar sustitutos óseos y técnicas de regeneración tisular guiada a fin de mejorar la integridad del proceso periodontal. La tasa de éxito conseguida con este tipo de implantes es similar a la obtenida con los implantes colocados convencionalmente tras la colocación de los mismos esperando el tiempo de cicatrización postexodoncia $(1,2,23-25)$.

Tal y como señala la literatura, esta técnica requiere de un meticuloso procedimiento quirúrgico, así como de cumplir unas correctas indicaciones clínicas, tales como la estabilidad primaria, ausencia de infección activa, suficiente espesor óseo crestal periimplantario como las más relevantes. Esta técnica no está exenta de complicaciones, siendo la más frecuente la infección postoperatoria que puede conducir a la pérdida del implante (3-7). La mayoría de los autores consideran que el requisito clínico de mayor importancia para conseguir éxito en este tipo de técnica se basa en la obtención de un alto torque de inserción del implante. Se considera que medidas superiores a $35 \mathrm{Nm}$ de inserción y valores superiores a 65 ISQ (Ostell) son adecuadas para obtener éxito en esta técnica $(12,15-17)$.

La mayoría de los autores revisados coinciden en evitar en la medida de lo posible levantar un colgajo mucoperióstico en la zona de colocación del implante, debido a la reabsorción ósea que ésta produce debido a la a alteración de la estabilidad y nutrición del hueso subyacente. Blanco y cols. (26), observaron, menor reabsorción de la tabla vestibular en implantes colocados con cirugía sin colgajo $(0,8 \mathrm{~mm})$ que con colgajo $(1,3 \mathrm{~mm})$. Por tanto, la desinserción del periostio tiene un papel vital en el modelado del alveolo después de una extracción debido a que puede originar una reabsorción osteoclástica aumentada. Hay que destacar el papel del grosor de la table vestibular a la hora de colocar implantes inmediatos postextracción. Chen y cols. $(17,18)$. Constataron reabsorciones marcadas incluso de 3 veces superiores en aquellos implantes inmediatos postextracción (IPOX) con paredes finas inferiores a $0,5 \mathrm{~mm}$. La predictibilidad del mantenimiento de la estética después de la colocación de estos implantes a sido poco estudiada y no tenemos suficientes datos.

\section{CONCLUSIONES}

La colocación de implantes inmediatos postextracción, sin ser una opción carente de inconvenientes, demues- 
tra ser una gran alternativa en gran parte de las situaciones clínicas, en las que es necesario la extracción dental.

Los implantes Naturactis, demostraron una gran estabilidad primaria de inserción, requisito indispensable para la colocación de IPOX así como factor clave en la predictibilidad de su éxito.

Los valores medidos en unidades ISQ con Ostell obtenidos, fueron altos; todos ellos con valores iniciales superiores a 63 ISQ.

No se observaron complicaciones inmediatas o diferidas en ningún caso a excepción de la pérdida de 4 implantes de forma temprana por infección del lecho alveolar. Ningún implante fracasó después de la carga protética.

Los estudios radiológicos iniciales, constatan un mantenimiento e integridad ósea crestal a 12 meses vista, en parte condicionado porque ningún caso refirió despegamiento mucoperióstico en su intervención.

Los datos evaluados a 6 meses vista son satisfactorios en base a la inserción, estabilidad y manejo del protocolo quirúrgico para la colocación del implante; sería conveniente ampliar el estudio con otros de mayor tamaño muestral y de forma comparativa con otros sistemas de implantes.

\section{BIBLIOGRAFÍA}

1. Peñarrocha M, Sanchís JM. Implante inmediato a la extracción. En: Peñarrocha M, ed. Implantología Oral. Barcelona: Ars Médica 2001. p. 85-93.

2. Bascones A, Frías MC, Bascones C. Implantes postextracción. JANO 2001;3:12-7.

3. Nail GA, Stein S, Korhi M, Waite DE. Evaluation of endosseous implants placed in fresh extraction sites in dogs (abstract 1906). J Dent Res 1990;69:347.

4. Wilson TG, Schenk R, Buser D, Cochran D. Implant placed in immediate extractions sites. A report of histologic and histometric analyses of human biopsies. Int J Oral Maxillofac Implants 1998;13:333-41.

5. Block MA, Kent JN. Placement of endosseous implants into tooth extractionssites. J Oral Maxillofac Surg 1991; 49:1269-76.
6. Albrektsson T, Branemark PI, Hansson HA, Lindstrom J. Osseointegrated titanium implants. Requirements for ensuring a long-lasting, direct bone-to-implant anchorage in man. Acta Orthopaedica Scandinavica 1981;52:155-70.

7. Amler $\mathrm{MH}$. The time sequence of tissue regeneration in human extraction wounds. Oral Surgery, Oral Medicine, Oral Pathology, Oral Radiology and Oral Endodontology 1969;27:309-18.

8. Anneroth G, Hedstrom KG, Kjellman O, Kondell PA, Nordenram $A$. Endosseus titanium implants in extraction sockets. An experimental study in monkeys. Inernational Journal of Oral Surgery 1985; 14:50-4.

9. Cochran DL, Hermann JS, Schenk RK, Higginbottom FL, Buser D. Biologic width around titanium implants. A histometric analysis of the implanto-gingival junction around unloaded and loaded nonsubmerged implants in the canine mandible. Journal of Periodontology 1997;68:186-98.

10. Covani U, Cornelini R, Barone A. Bucco-lingual bone remodeling around implants placed into immediate extraction sockets: a case series. Journal of Periodontology 2003;74:268-73.

11. Botticelli D, Berglundh T, Buser D, Lindhe J. The jumping distance revisited: An experimental study in the dog. Clinical Oral Implant Research 2003b; 14:35-42.

12. Botticelli D, Berglundh T, Lindhe J. Hard-tissue alterations following immediate implant placement in extraction sites.Journal of Clinical Periodontology 2004;31:820-8.

13. Botticelli D, Persson LG, Lindhe J, Berglundh T. (2006) Bone tissue formation adjacent to implants placed in fresh extraction sockets: an experimental study in dogs. Clinical Oral Implant Research 2006;17:351-8.

14. Botticelli D, Berglundh T, Buser D, Lindhe J. Appositional bone formation in marginal defects at implants. Clinical Oral Implant Research 2003a;14;1-9.

15. Cardaropoli D, Debernardi C, Cardaropoli G. Immediate placement of implant into impacted maxillary canine extraction socket. Int The International Journal of Periodontics and Restorative Dentistry 2007; 27:71-7.

16. Cardaropoli G, Wennstrom JL, Lekholm U. Peri-implant bone alterations in relation to inter-unit distances. A 3- 
year retrospective study. Clinical Oral Implant Research 2003; 14:430-6.

17. Chen ST, Darby IB, Reynolds EC. A prospective clinical study of non-submerged immediate implants: clinical outcomes and esthetic results. Clinical Oral Implant Research 2007;18:552-62.

18. Chen ST, Wilson TG Jr, Hammerle CH. Immediate or early placement of implants following tooth extraction: review of biologic basis, clinical procedures, and outcomes. International Journal of Oral and Maxillofacial Implants 2004; 19 Suppl:12-25.

19. Araujo MG, Sukekava F, Wennstrom JL, Lindhe J. Ridge alterations following implant placement in fresh extraction sockets: an experimental study in the dog. Journal of Clinical Periodontologyy 2005;32:645-52.

20. Araujo MG, Sukekava F, Wennstrom JL, Lindhe J. Tissue modeling following implant placement in fresh extraction sockets. Clinical Oral Implant Research 2006a; 17:615-24.

21. Araujo MG, Wennstrom JL, Lindhe J. Modeling of the buccal and lingual bone walls of fresh extraction sites following implant installation. Clinical Oral Implant Research 2006b;17:606-14.

22. Boyne PJ. (1966) Osseous repair of the postextraction alveolus in man. Oral Surgery, Oral Medicine, Oral
Pathology, Oral Radiology and Oral Endodontology 21 , 805-813.

23. Carmagnola D, Adriaens P, Berglundh T. Healing of human extraction sockets filled with Bio-Oss. Clinical Oral Implant Research 2003;14:137-43.

24. Dies F, Etienne D, Abboud NB, Ouhayoun JP. Bone regeneration in extraction sites after immediate placement of an e-PTFE membrane with or without a biomaterial. A report on 12 consecutive cases. Clinical Oral Implant Research 1996;7:277-85.

25. Cornelini R, Scarano A, Covani U, Petrone G, Piattelli A. Immediate one-stage postextraction implant: A human clinical an histologic case report. Int J Oral Maxillofac Implants 2000;15:432-7.

26. Blanco J, Nunez V, Aracil L, Muñoz F, Ramos I. Ridge alterations following immediate implant placement in the dog: flap versus flapless surgery. Journal of Clinical Periodontology 2008;35:640-8.

\section{CORRESPONDENCIA}

Jorge Ripollés de Ramón

Platerías, 1

28016 Madrid

Correo electrónico: jorgeripolles@hotmail.com 